\title{
Oleanolic acid mediated the proliferation and invasion of U251 glioma cells and promoted their apoptosis through the IKK- $\beta$, MAPK3, and MAPK4 signaling pathway
}

Jinxiang Huang

Changzheng Hospital: Shanghai Changzheng Hospital

Shengnan Lin

Sanming First Hospital and First Hospital of Sanming Affiliated to Fujian Medical University

Feng Zhu

Sanming First Hospital and First Hospital of Sanming Affiliated to Fujian Medical University

Dengsheng Chen

Sanming First Hospital and First Hospital of Sanming Affiliated to Fujian Medical University

Fang Huang

Sanming First Hospital and First Hospital of Sanming Affiliated to Fujian Medical University luning xu ( $\nabla$ xlning@fjmu.edu.cn )

Sanming First Hospital and First Hospital of Sanming Affiliated to Fujian Medical University https://orcid.org/0000-0003-3160-8572

\section{Research Article}

Keywords: Glioma, Oleanolic acid, IKK- $\beta$, MAPK3, MAPK4

Posted Date: July 26th, 2021

DOI: https://doi.org/10.21203/rs.3.rs-705871/v1

License: (a) (i) This work is licensed under a Creative Commons Attribution 4.0 International License. Read Full License 


\section{Abstract}

Object

To investigate the effects of Oleanolic acid (OA) on proliferation, apoptosis, migration, and invasion of human glioma cell U251, as well as IKK- $\beta$ and MAPK signaling pathways.

Methods

The binding of OA to IKK- $\beta$ and MAPK signaling pathway essential proteins IKK- $\beta$, MAPK3, and MAPK4 was analyzed by molecular docking technique. U251 cells were treated with different concentrations of OA. The proliferation and apoptosis rates of U251 cells were detected by CCK-8 assay, MTT assay, cell cloning assay, and Annexin $\otimes$ FITC/PI double staining assay. Transwell chamber assay was used to detect migration and invasion of U251 cells. Finally, Western blotting was used to detect the protein expression levels of IKK- $\beta$, MAPK3, and MAPK4 in U251 cells treated with OA.

Results

The results of molecular docking showed that OA could stably bind to IKK- $\beta$, MAPK3, and MAPK4 proteins. OA could not only effectively inhibit the proliferation and induce apoptosis of U251 cells $(\mathrm{P}<$ $0.05)$, but also significantly inhibit the invasion of U251 cells $(P<0.05)$. Western blot assay confirmed that OA could dramatically inhibit the protein expression levels of IKK- $\beta$, MAPK3, and MAPK4 in U251 cells $(P<0.05)$.

Conclusions

OA may inhibit the proliferation, migration, and invasion of glioma U251 cells by binding key molecules of the IKK- $\beta$ signaling pathway and essential target proteins of MAPK3 and MAPK4 in the MAPK signaling pathway.

\section{Background}

Glioma is currently the most aggressive and fatal human intracranial primary malignant brain tumor ${ }^{[1]}$. Because of the specific and remote location of these tumors, removal of glioma is difficult, and thus glioma is associated with high morbidity and mortality rates ${ }^{[2]}$. The clinical treatment of glioma includes surgery, radiotherapy, and chemotherapy drugs, but the prognosis in patients remains unsatisfactory ${ }^{[3]}$ and the 5 -year survival rate of glioma is less than $10 \%$. Therefore, identifying anti-tumor drugs with good curative effects, minor side effects, and high safety is critical. At present, targeted therapy has become a new research hotspot. Compared with traditional medicines, small molecule targeted drugs have increased efficacy in targeting tumors and show advantages such as specificity, accurate targeting, good tolerance, mild toxicity, and passing the blood-brain barrier ${ }^{[4]}$. 
Oleanolic acid $(\mathrm{OA})$ is a type of natural pentacyclic triterpenoid compound that is present in many plants such as Hedyotis officinalis, Ligustrum lucidum, papaya, loquat leaves, olive, and others. OA has many pharmacological effects, such as protecting the liver, lowering lipids, lowering glucose, and enhancing immunity. Studies have shown that OA can induce apoptosis in a variety of tumor cells, such as lung cancer, pancreatic cancer ${ }^{[5]}$, cervical cancer ${ }^{[6]}$, and ovarian cancer cells ${ }^{[7]}$, and many other types of tumor cells that have anti-proliferation effect. Currently, OA is widely used in clinical medicine for the treatment of acute and chronic hepatitis and as an anti-cancer adjuvant drug ${ }^{[8]}$.

In this study, we investigated the effects of OA on proliferation, migration, and invasion of glioma cells and the IKK- $\beta$, MAPK3, and MAPK4 proteins, with the aim of providing experimental basis for the clinical treatment of glioma patients with $\mathrm{OA}$.

\section{Materials And Methods 1.1 Materials}

Oleanolic acid (Lot: AF9071301), its molecular formula is $\mathrm{C} 30 \mathrm{H} 4803$, molecular weight is 456.70 , purchased from Chengdu Alfa Biotechnology Co., Lid. Purity of the compound used in the present study was shown to be higher than $98 \%$ by HPLC; The human glioma cell line U251 was purchased from the Cell Bank of Shanghai Academy of Sciences; DMEM was purchased from Hyclone, USA; fetal bovine serum was from GIBCO. The CCK-8 kit, PBS, $0.25 \%$ trypsin digestion solution, trypsin EDTA digestion solution, penicillin-streptomycin, and electrophoresis buffer were all purchased from Solarbio. The RIPA tissue cell rapid lysate and BCA protein quantification kits were purchased from Beyotime. HRP-labeled secondary antibody and the Annexin V-FITC cell apoptosis detection kit were purchased from Biyuntian Biotechnology Co., Ltd. Formaldehyde was purchased from Sinopharm Chemical Reagent Co., Ltd. Matrigel was purchased from Corning Company. IKK $\beta$ and MAPK4 antibodies were purchased from Proteintech; MAPK3 antibody was purchased from Affinity; and GAPDH antibody was purchased from CST. The Type $311 \mathrm{CO} 2$ constant temperature incubator was purchased from Thermo Company. The AMR-100 microplate reader was purchased from Hangzhou Aosheng. The DMIL LED inverted fluorescence microscope was purchased from Leica. The Mini-PROTEAN 3 cell electrophoresis instrument was purchased from BIO-RAD. The Tanon-5200 imaging system was purchased from Tanon. The CytoFLEX flow cytometer was purchased from Beckman Coulter. The Transwell Chamber model 3422 was purchased from COSTAR Corporation.

\subsection{Molecular docking}

IKK- $\beta$ (PDB ID: 4KIK), MAPK3 (PDB ID: 2QNJ) and MAPK4 (PDB ID: 5ES1) data were downloaded from the RSCB PDB database (http://www.rcsb.org/pdb/home/home.do). The 3D structures of the target proteins were saved as a PDB format file. The mol2 format file of OA was downloaded from the ZINC website (http://zinc.docking.org/). The PyMOL software was used to remove water, phosphate, and other irrelevant ions from the protein. The target protein receptor and ligand were separated, and the ligand 
molecules were extracted. The pre-processed target protein and the original ligand were saved as PDB format files. AutoDockTools 1.5.6 software was used to set the size of Grid Box and box, search for the parameters of protein active site (active port), convert the active small molecules, pretreated target protein, and original ligand into pdbqt format file, run AutoDock Tools 1.5.6 software to perform Autogrid and Docking operations, and finally use Open Babel GUI and PyMOL software to analyze the docking results visually.

\subsection{Cell culture}

Human glioma U251 cells were cultured in DMEM high glucose medium containing $10 \%$ fetal bovine serum and $1 \%$ penicillin-streptomycin in a $5 \% \mathrm{CO}_{2}$ incubator at $37^{\circ} \mathrm{C}$. Cells in logarithmic growth phase were used in experiments.

\subsection{Cell viability assay}

Cell viability of U251 cells was measured using a CCK-8 assay. Cells were plated in a 96-well plate with $3 \times 103$ cells/well in $100 \mu \mathrm{L}$ per well and cultured overnight. Cells were treated with $\mathrm{OA}(0,2.74,5.47,10.95$, $21.90,32.84,43.79,54.74,109.48$, and $218.96 \mu \mathrm{mol} / \mathrm{L})$, with three wells for each treatment group, for $8 \mathrm{~h}$. Next, $10 \mu \mathrm{L}$ of CCK-8 solution was added to each well, and cells were incubated for $4 \mathrm{~h}$. The OD value of each well was measured at $450 \mathrm{~nm}$ with an enzyme-linked immunoassay. The relative proliferation inhibition rate was calculated as (\%) $=[1-(O D$ value of each drug group-OD value of the blank group) / (OD value of the control group-OD value of the blank group)] $\times 100 \%$.

\subsection{MTT assay}

Cells were plated in 96-well culture plates at $1-5 \times 10^{4}$ cells $/ \mathrm{mL}$ and cultured overnight. Cells were then treated with $\mathrm{OA}(0,21.90,43.79,109.48,218.96,437.92,1094.81,2189.62,4379.24$ and 10948.11 $\mu \mathrm{mol} / \mathrm{L}$ ), with three wells for each treatment group, and cultured for an additional $24 \mathrm{~h}$. Next, $20 \mu \mathrm{L}$ of MTT solution ( $5 \mathrm{mg} / \mathrm{mL}$ ) was added to each well, and cells were incubated for $4 \mathrm{~h}$. The supernatant was then discarded. DMSO $(150 \mu \mathrm{L})$ was added to each well and the plates were shaken for $10 \mathrm{~min}$. Absorbance at $490 \mathrm{~nm}$ was measured with an enzyme plate analyzer. The proliferation inhibition rate of each group was calculated.

\subsection{Colony formation assay}

Cells were inoculated in a $6 \mathrm{~cm}$ dish at $1 \times 10^{3}$ cells per dish and then cultured for 2 to 3 weeks. The culture was discontinued when the cells formed visible clones. The supernatant was discarded and the plates were washed with PBS; cells were fixed with $4 \%$ paraformaldehyde for $15 \mathrm{~min}$. The fixed solution was discarded, and cells were stained with crystal violet staining solution for 10-30 min. The staining solution was removed and plates were air-dried. The number of clones was calculated using a microscope, and the rate of clone formation was determined as $=$ (number of clones/number of inoculated cells) $\times 100 \%$.

\subsection{Transwell assays}


Transfected cells were cultured in serum-free medium for $24 \mathrm{~h}$ before the experiment. Matrigel mixed with serum-free medium $(1: 2 ; 80 \mu \mathrm{L})$ was added to a Transwell chamber, and the chamber was incubated at $37^{\circ} \mathrm{C}$ for $30 \mathrm{~min}$ to solidify. Cells $(300 \mu \mathrm{L}, 3 \times 105 \mathrm{cells} / \mathrm{mL})$ were added to the top chamber of the Transwell chamber and $700 \mu \mathrm{L}$ of $10 \%$ FBS-containing complete culture medium was added to the bottom chambers. Cells were cultured for $24 \mathrm{~h}$. Next, $4 \%$ formaldehyde solution was added to each well and cells were fixed at room temperature for $10 \mathrm{~min}$. The fixing solution was removed and the cells were washed by PBS, followed by staining with $1 \mathrm{ml}$ of $0.5 \%$ crystal violet solution for $30 \mathrm{~min}$. Samples were washed three times with PBS and air-dried. The cells were observed and photographed under a microscope (at 400x), and the number of invaded cells in each group was counted.

\subsection{Flow cytometry assay}

Cells were harvested ( $5 \times 10^{4}$ to $1 \times 10^{5}$ cells) and resuspended in PBS. Next, $195 \mu \mathrm{L}$ Annexin V-FITC binding solution was added to cells, followed by $10 \mu \mathrm{L}$ Annexin V-FITC and $5 \mu \mathrm{L}$ propidium iodide staining solution. The samples were mixed and incubated in the dark at room temperature for $15 \mathrm{~min}$ and then placed in an ice bath. A sample without Annexin V-FITC and PI was used as a negative control. The apoptosis rate of each group was detected by flow cytometry.

\subsection{Western blotting assay}

Cells were lysed with RIPA rapid lysis buffer and the BCA protein quantitative method was used to determine protein concentration. After denaturation, protein samples were separated by $10 \%$ SDS-PAGE gel electrophoresis and transferred to a PVDF membrane. The membrane was blocked with $5 \%$ skimmed milk powder at room temperature for $1 \mathrm{~h}$ and then incubated with the following primary antibodies overnight at $4^{\circ} \mathrm{C}$ : IKK $\beta$ antibody (1:600), MAPK3 antibody (1:1000), MAPK4 antibody (1:400), and GAPDH antibody (1:2000). After three washes in TBST for $5 \mathrm{~min}$, the membrane was incubated with HRP-labeled secondary antibody $(1: 1000)$ at $37^{\circ} \mathrm{C}$ for $1 \mathrm{~h}$ and then washed with TBST three times. The membrane was developed using the ECL chemiluminescence method and the protein bands were visualized using a chemiluminescence imaging system. The protein bands were analyzed by the Tanon- 5200 image analysis system with GAPDH as the internal reference.

\subsection{Statistical analysis}

GraphPad Prism 5.0 software was used for image processing. The SPSS 21.0 software was used for statistical analysis. Measurement data are expressed as $\nabla x \pm s$, and the comparison of multiple groups was performed by the one-way analysis of variance (ANOVA) method. $\mathrm{P}<0.05$ indicated statistical significance.

\section{Results}

\subsection{OA shows binding activity with IKK- $\beta$, MAPK3, and MAPK4}


The interaction model of OA with IKK- $\beta$, MAPK3, and MAPK4 was examined through molecular docking. The docking results are shown in Table 1 and Fig. 1. Studies have shown that a lower binding energy required for the binding of the ligand and the receptor is associated with a more stable binding conformation and higher binding possibility. A binding energy less than 0 indicates that the ligand and receptor can spontaneously bind. We found that the binding energy of OA with IKK- $\beta$, MAPK3, and MAPK4 was $-5 \mathrm{KJ} / \mathrm{mol}$ through hydrogen bonds with ASP-484, TYR-134, ILE-62, LYS-193, GLU-81, and ASP-187 in IKK- $\beta$, MAPK3, and MAPK4 kinases, indicating that OA has an excellent binding activity with the three target proteins.

\subsection{OA inhibits the proliferation of U251 cells}

The CCK-8 assay results showed that OA inhibited U251 glioma cells proliferation. Concentrations of $109.48 \mu \mathrm{mol} / \mathrm{L}$ induced $100 \%$ inhibition. Significant inhibition of proliferation was seen at concentrations at low as $54.74 \mu \mathrm{mol} / \mathrm{L}$. (Fig. 2). There were statistically significant differences in the inhibition rates among the different groups $(P<0.05)$. These results suggested that $O A$ inhibits the proliferation of U251 glioma cells in a dose-dependent manner.

Table 1 Docking results of OA with IKK- $\beta$, MAPK3, and MAPK4 proteins

\begin{tabular}{llll} 
Chemical compound & Target & PDB_ID & Binding energy $\square \mathrm{KJ} / \mathrm{mol}$ \\
Oleanolic Acid & IKK- $\beta$ & $4 \mathrm{KIK}$ & -6.1 \\
\cline { 2 - 4 } & MAPK3 & 2QNJ & -7.11 \\
\hline MAPK4 & $5 \mathrm{ES} 1$ & -6.64
\end{tabular}

The MTT assay results showed that OA inhibited U251 glioma cells proliferation. Concentrations of $437.92 \mu \mathrm{mol} / \mathrm{L}$ induced $91 \%$ inhibition. Significant inhibition of proliferation was seen at concentrations at low as $43.79 \mu \mathrm{mol} / \mathrm{L}$. (Fig. 3). The difference in the inhibition rate between the different concentration treatment groups was statistically significant $(P<0.05)$. These results suggest that $\mathrm{OA}$ significantly inhibits the proliferation of U251 cells in a concentration-dependent manner.

\subsection{Cell clone assay to detect cell proliferation}

We next performed cell clone assays to evaluate the effect of OA on the colony formation ability of U251 glioma cells. The number of cell clones $(41 \pm 2)$ in the OA group was significantly reduced compared with the number in the control group $(124 \pm 7)(P<0.05)$ (Fig. 4). These results confirmed that OA treatment significantly decreased the colony formation ability of U251 glioma cells.

\subsection{Cell invasion was detected by Transwell chamber assay}

Transwell experiments showed that the number of invaded cells in the OA treatment group ( $34 \pm 4)$ was significantly reduced compared with the control group $(74 \pm 5)(P<0.05)($ Fig. 5). These results indicated that OA substantially inhibits the invasion ability of human glioma U251 cells.

\subsection{OA promotes apoptosis of human glioma U251 cells}


We next evaluated apoptosis using Annexin V-FITC/PI double staining and flow cytometry. The results showed that the apoptotic rate of U251 cells after OA treatment was $25.27 \pm 1.11 \%$, while the apoptotic rate of the control group was $4.39 \pm 0.22 \%$ (Fig. 6). The apoptotic rate increased significantly after $\mathrm{OA}$ treatment, and the difference was statistically significant $(P<0.05)$. These results showed that $O A$ promotes the apoptosis of human glioma U251 cells.

\subsection{OA downregulates the expression of IKK- $\beta$, MAPK3, and MAPK4 protein}

We next used western blot analysis to evaluate the expressions of IKK- $\beta$, MAPK3, and MAPK4 proteins in U251 glioma cells treated with OA. After OA treatment of U251 glioma cells, the expression levels of IKK- $\beta$, MAPK3, and MAPK4 protein were reduced compared with control cells, and the differences were statistically significant $(P<0.05)$ (Fig. 7). These results indicate that $O A$ treatment downregulates the expressions of IKK- $\beta$, MAPK3, and MAPK4 proteins in U251 glioma cells.

\section{Discussion}

Glioma is the most common malignant tumor among all intracranial malignancies ${ }^{[9]}$ and is a complex tumor lesion that is characterized by invasiveness and a high recurrence rate. Despite the availability of treatments, such as early surgical resection, radiation, or chemotherapy, the prognosis of patients with glioma is poor. In addition to the poor therapeutic effect of chemotherapy, early radiotherapy, and late adjuvant therapy on malignant glioma, these treatments also have toxic side effects. Therefore, identifying new therapeutic agents to improve the prognosis of patients with malignant glioma is critical $[10,11]$.

Glioma progression involves abnormal gene expression, including overexpression of oncogenes, mutations or deletions of tumor suppressor genes, and abnormal changes in signaling pathways and other cellular processes. To overcome the limitations of the existing treatment methods for malignant glioma, many new treatment methods such as molecular targeted therapy, immunotherapy, gene therapy, stem cell therapy, and nanotechnology have begun to appear in preclinical and clinical research. In recent years, traditional Chinese medicine has shown significant value in the treatment of human glioma. $\mathrm{Bi}^{[12]}$ found that Paris polyphylla saponin reduced the viability of U251 cells by inhibiting the expression of ARA1 and ARA3, leading to inhibited phosphorylation of Akt and p44/42 MAPK and induced apoptosis

and cell cycle arrest. Liu ${ }^{[13]}$ showed that berberine inhibited the proliferation of glioma cells by interfering with wild-type and mutant p53.

$\mathrm{OA}$ is an effective anti-tumor drug that inhibits glioma cells with a variety of malignant phenotypes. Studies have shown that $\mathrm{OA}$ and its derivatives have no cytotoxicity to normal human cells ${ }^{[14]}$ and it inhibits tumor development in various malignant tumors through multiple methods and pathways. In vivo experiments showed that OA significantly reduced the size and quality of tumors, and in vitro experiments 
revealed that $O A$ has a significant inhibitory effect on cell migration, cell viability and proliferation ability $[15,16]$. OA has a sensitizing effect on radiotherapy of $\mathrm{C} 6$ cell-bearing rats ${ }^{[17]}$. The pathway by which OA inhibits cell processes varies in different tumor cells, and its anti-tumor effect is related to the inhibition of intracellular signaling pathways such as STAT3, VEGF, MAPK, ERK, JNK, Akt, mTOR, and NF- $k B$ pathways. In this study, molecular docking technology showed that the binding energies of OA and IKK- $\beta$, MAPK3, and MAPK4 proteins were all less than $-5 \mathrm{kcal} / \mathrm{mol}$, indicating that OA had a good binding activity with the three proteins. We speculate that $O A$ may inhibit glioma proliferation by downregulating IKK- $\beta$, MAPK3, and MAPK4 proteins.

To further study the effect of OA on glioma, we performed in vitro experiments. CCK-8 and MTT assays revealed that the proliferation inhibition rate of cells increased in response to OA in a dose-dependent manner. Colony formation assays showed that the number of U251 cells decreased significantly after OA treatment. Together these results indicated that OA inhibits the proliferation of U251 cells.

Invasion is a critical biological feature of malignant tumors. It is the process by which tumor cells penetrate the blood vessel wall and enter the circulatory system. Transwell assays showed that the number of invaded U251 cells treated with OA was significantly reduced compared with the control group, suggesting that $O A$ inhibits the invasion of U251 cells.

Apoptosis is a process in which cells undergo programmed death through gene regulation. Many tumor suppressor genes inhibit tumor development through inducing apoptosis, and apoptosis induction is one of the main mechanisms of current tumor treatments. Flow cytometry showed that the apoptosis rate of cells treated with OA was significantly increased, indicating OA has a promoting effect on cell apoptosis. Together these results show that OA inhibits the proliferation and invasion of glioma U251 cells and promotes glioma cell apoptosis, thereby exhibiting anti-cancer effects in glioma.

The IKK and MAPK pathways play an essential role in the proliferation, migration, and invasion of glioma cells. The overexpression of IKK- $\beta$ is closely related to the occurrence of a variety of malignant tumors, and inhibition of IKK- $\beta$ in tumor cells results in specific anti-tumor effects ${ }^{[18-20]}$. Recent studies found a close relationship between malignant tumors and chronic inflammation. IKK- $\beta$ activation helps promote the occurrence of cancer and inflammatory lesions, and inflammation can inhibit cell apoptosis through the IKK- $\beta$ pathway, thus enabling the development malignant tumors. The specific inhibition of IKK- $\beta$ provides a new strategy and approach for the prevention and targeted treatment of inflammationassociated tumors. Activation of the IKK/NF-KB pathway induces tumor cells to proliferate and migrate and inhibits apoptosis ${ }^{[21]}$. Nan et al. demonstrated that miR-451 inhibited glioma invasion and migration in vivo and in vitro by regulating IKK- $\beta^{[22]}$. Wang et al. showed that TGF $\beta 1$ regulates TNF- $\kappa B$ activity induced by TNF- $a$ in glioma cells through the PP1A/IKK- $\beta$ pathway and promotes the progression and development of gliomas ${ }^{[23]}$.

MAPK plays a vital role in regulating cell growth, division, differentiation, and apoptosis. The MAPK pathway participates in the regulation of cancer cells through roles in proliferation, differentiation, and 
apoptosis, and other critical biological responses ${ }^{[24,25]}$. This pathway mainly regulates cell proliferation and apoptosis, among which abnormalities in the cell growth stage or in a particular link in vivo may cause cell growth control and tumorigenesis. Blocking the MAPK pathway can inhibit the formation of tumors. Recent studies showed that the MAPK signaling pathway has a series of active metastases in glioma and other malignant tumor tissues, and MAPK directly mediates the anti-tumor effects of a variety of drugs. One study showed that miR-326 inhibits the malignant biological behavior of glioma cells through the MAPK signaling pathway ${ }^{[26]}$. MAPK3 and MAPK4 are members of the MAPK pathway. Yang et al. showed that miR-127-3p inhibits the proliferation of U251 glioma cells by down-regulating the target gene MAPK4 ${ }^{[27]}$. Some researchers also reported that a new MAPK3 and MAPK4 double-molecule inhibitor PCC0208017 reduced glioma cell migration, glioma cell invasion, and angiogenesis and may be a promising lead compound for the treatment of glioma ${ }^{[28]}$.

The studies described above showed that the IKK- $\beta$ /MAPK3/MAPK4 signaling pathway plays an essential role in the development of glioma. In this study, our western blot analysis showed that the protein expression levels of IKK- $\beta$, MAPK3, and MAPK4 were suppressed in glioma cells treated with OA. We speculate that OA may inhibit IKK- $\beta$, MAPK3, and MAPK4 protein expression by directly binding to the proteins.

In summary, we found that OA inhibits the proliferation and invasion of U251 glioma cells and promotes cell apoptosis. We further showed that OA inhibited the IKK- $\beta$ /MAPK3/MAPK4 signaling pathway in glioma cells. These findings suggest that OA may have a good application prospect in the clinical treatment of glioma and provides ideas for the development and study of tumor drugs. Our research group will further research gene sequencing and other aspects. The treatment of human glioma with OA needs to be further studied in vivo.

\section{Declarations}

\section{Acknowledgments}

This work was supported by Natural Science Foundation of Fujian Province (2018J0105, China).

\section{Author contributions}

Luning Xu designed the research. Jinxiang Huang, Shengnan Lin, Feng Zhu, Dengsheng Chen, and Fang Huang performed experiments. Luning $\mathrm{Xu}$, Jinxiang Huang, and Shengnan Lin performed data analyses. Luning $\mathrm{Xu}$ and Jinxiang Huang wrote the paper.

\section{Funding}

This work was supported by Natural Science Foundation of Fujian Province (2018J0105, China).

\section{Competing interests}


The authors declare that they have no competing interests.

\section{Availability of data and materials}

All data generated or analysed during this study are included in this published article.

\section{Consent for publication}

Not applicable.

\section{Ethics approval and consent to participate}

Not applicable.

\section{References}

1. Goudarzi Kaveh M, Espinoza Jaime A, Guo Min B, Jiri N, Monica, Lindström Mikael S, et al. Reduced Expression of PROX1 Transitions Glioblastoma Cells into a Mesenchymal Gene Expression Subtype[J]. Cancer research,2018,78(20):5901-5916.

2. Diao T, Zhang Y, Lu W, Min Q. Inhibitory effect of total flavonoids from hawthorn leaves on glioma U87 cells [J]. Chinese Journal of Pharmacology. 2019;35(10):1448-52.

3. Christopher M, Jackson J, Choi M, Lim. Mechanisms of immunotherapy resistance: lessons from glioblastoma [J]. Nature Immunology,2019,20(14):1100-1109.

4. Andrew DJ, Pearson R, Herold R, Rousseau C, Copland B, Bradley-Garelik D, Binner, et al. Implementation of mechanism of action biology-driven early drug development for children with cancer[J]. Eur J Cancer. 2016;62:124-31.

5. Xiaojing Shen H, Zhao L, Zhao. Weiwei Jiang. Research progress of oleanolic acid [J]. Guangzhou Chemical Industry,2019,47(24).

6. Xiaofei Jiang M, Shi M, Sui Y, Yuan S, Zhang Q, Xia, et al. Oleanolic acid inhibits cervical cancer Hela cell proliferation through modulation of the ACSL4 ferroptosis signaling pathway[J]. Biochem Biophys Res Commun. 2021;545:124e131.

7. Du G, Zhang Y. Xiaohui Hu. Effects of Olenolic acid on cell proliferation and metastasis of human ovarian cancer SKOV3 cells [J]. China Pharmacy,2020,31(10):1190-1197.

8. Tang S, Gao D, Zhao T, Zhou J, Zhao X. An evaluation of the anti-tumor efficacy of oleanolic acidloaded PEGylated liposomes[J]. Nanotechnology. 2013;24(23):235102.

9. Quinn T, Ostrom H, Gittleman J, Xu C, Kromer Y, Wolinsky C, Kruchko, et al. CBTRUS Statistical Report: Primary Brain and Other Central Nervous System Tumors Diagnosed in the United States in 2009-2013[J]. Neuro-oncology,2016,18(suppl_5): v1-v75. 
10. Ping Liu J, Yu X, Tian J, Chang Y, Zhang R, Zhang, et al. The effect of downregulation of Stathmin gene on biological behaviors of U373 and U87-MG glioblastoma cells[J]. Biol Res. 2018;51(11):492507.

11. Katharina Seystahl W, Wick M, Weller. Therapeutic options in recurrent glioblastoma-An update[J]. Critical Reviews in Oncology / Hematology,2016,99: 389-408.

12. Linlin Bi Y, Liu Q, Yang X, Zhou H, Li Y, Liu, et al. Paris saponin H inhibits the proliferation of glioma cells through the $A 1$ and $A 3$ adenosine receptor-mediated pathway[J]. International Journal of Molecular Medicine,2021,47(4): 1.

13. Ziqiang Liu Y, Chen H, Gao W, Xu C, Zhang J, Lai, et al. Berberine Inhibits Cell Proliferation by Interfering with Wild-Type and Mutant P53 in Human Glioma Cells[J]. OncoTargets and therapy,2020,13: 12151-12162.

14. Guo G, Yao W, Zhang Q. Yongli Bo. Oleanolic Acid Suppresses Migration and Invasion of Malignant Glioma Cells by Inactivating MAPK/ERK Signaling Pathway[J]. PLOS ONE,2013,8(8): e72079.

15. Edathara PM, Chintalapally S, Makani VKrishnaK, Pant C, Yerramsetty S, Manohar D, Rao, et al. Inhibitory Role of Oleanolic Acid and Esculetin in Hela Cells Involve Multiple Signaling Pathways[J]. Gene,2020,771: 145370e145370.

16. Xiaofei Jiang M, Shi M, Sui Y, Yuan S, Zhang Q, Xia, et al. Oleanolic acid inhibits cervical cancer Hela cell proliferation through modulation of the ACSL4 ferroptosis signaling pathway[J]. Biochem Biophys Res Commun. 2021;545:124e131.

17. YZ 1, H Xu, Wang H, Yu W, Zhao X, Xue Y. Fluorine-18-deoxyglucose positron emission tomography/computed tomography with Ki67 and GLUT-1 immunohistochemistry for evaluation of the radiosensitization effect of oleanolic acid on $\mathrm{C} 6$ rat gliomas[J]. Nuclear medicine communications,2015,36(1):21e27.

18. Zhu Y, Zhou Y, Zhou X, Guo Y, Huang D, Zhang J, et al. S100A4 suppresses can-cer stem cell proliferation via interaction with the IKK/NF-kappaB signaling pathway[J]. BMC Cancer. 2018;18(1):763.

19. Nikee Awasthee V, Rai S, Chava P, Nallasamy, Ajaikumar B, Kunnumakkara A, Bishayee, et al. Targeting ІкарраB kinases for cancer therapy[J]. Seminars in Cancer Biology,2019,56:12e24.

20. Jack A, Prescott, Simon J. Cook. Targeting IKK $\beta$ in Cancer: Challenges and Opportunities for the Therapeutic Utilisation of IKKß Inhibitors[J]. Cells,2018,7(9): e115.

21. Amanda L, Rinkenbaugh PC, Cogswell B, Calamini DE, Dunn, Anders I, Persson, William A, Weiss, et al. IKK/NF-KB signaling contributes to glioblastoma stem cell maintenance[J]. Oncotarget, 2016, 7(43).

22. Nanyang. Study on the mechanism of miR-451 regulating NF-KB/Snail signaling pathway by targeting IKK- $\beta$ to inhibit glioma invasion and migration [D]. Tianjin Medical University; 2017.

23. Huizhi Wang Z, Chen S, Wang X, Gao M, Qian W, Qiu, et al. TGF 31 -induced beta-site APP-cleaving enzyme 2 upregulation promotes tumorigenesis through the NF-KB signalling pathway in human gliomas[J]. Molecular Oncology; 2020. 
24. Khaled A, Selim H, Abdelrasoul M, Aboelmagd, Ahmed M, Tawila. The role of the MAPK signaling, topoisomerase and dietary bioac-tives in controlling cancer incidence[J]. Diseases. 2017;5(2):13.

25. Nan Tang J, Chang H-C, Lu Z, Zhuang H-L, Cheng J-X. Shi, et al. Rhein induces apoptosis and autophagy in human and rat glioma cells and mediates cell differentiation by ERK inhibition[J]. Microbial Pathogene-sis. 2017;113:168-75.

26. Jingxu Zhou T, Xu Y, Qin YR, Wang H, Zhang X, et al. MicroRNA-326 Functions as a Tumor Suppressor in Glioma by Targeting the Nin One Binding Protein (NOB1) [J]. PLoS ONE. 2013;8(7):e68469.

27. Yan yang. Han meiwen, Wang yingrui. mechanism of miR-127-3p inhibiting glioma proliferation by downregulating MAPK4 [J]. stroke nervous diseases. 2018;25(04):435-9 + 470.

28. Li F, Liu Z, Sun H, Li C, Wang W, Ye L, et al. PCC0208017, a novel small-molecule inhibitor of MARK3/MARK4, suppresses glioma progression invitro and in vivo [J]. Acta Pharmaceutica Sinica B. 2020;10(2):289-300.

\section{Figures}

A Oleanolic Acid-IKK $\beta$

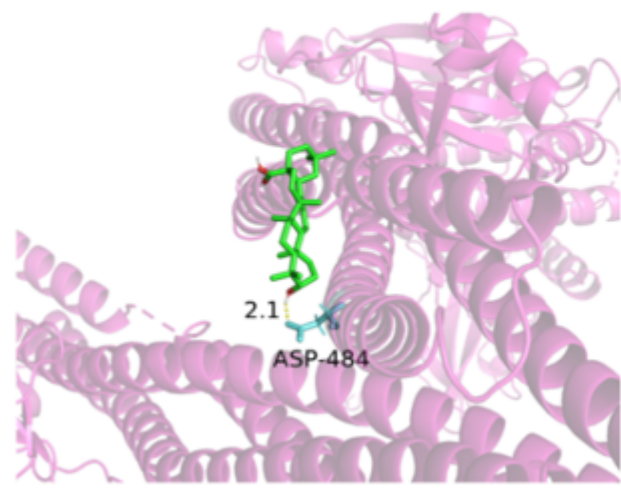

B Oleanolic Acid-MAPK3

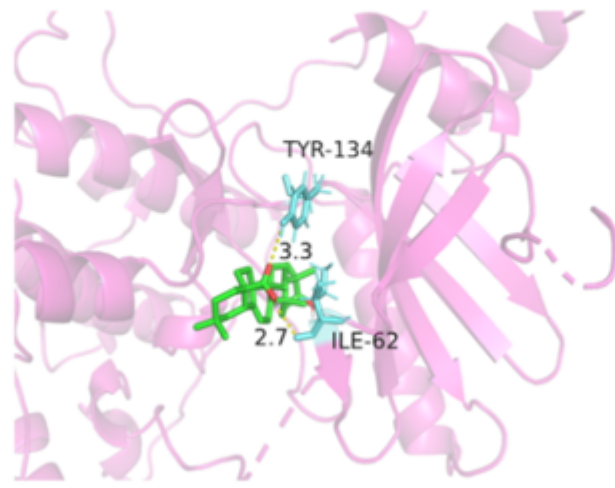

C Oleanolic Acid-MAPK4

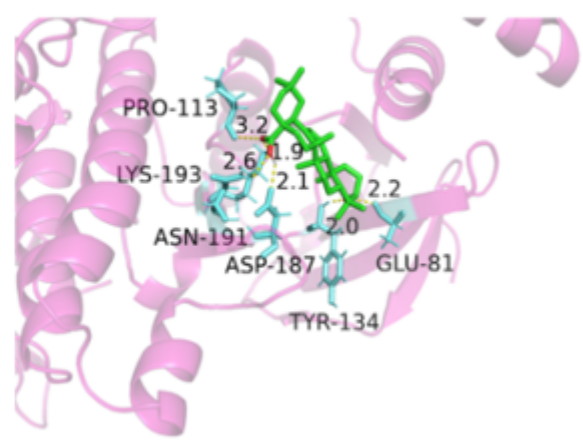

\section{Figure 1}

Molecular docking diagram of OA and IKK- $\beta$, MAPK3, and MAPK4 


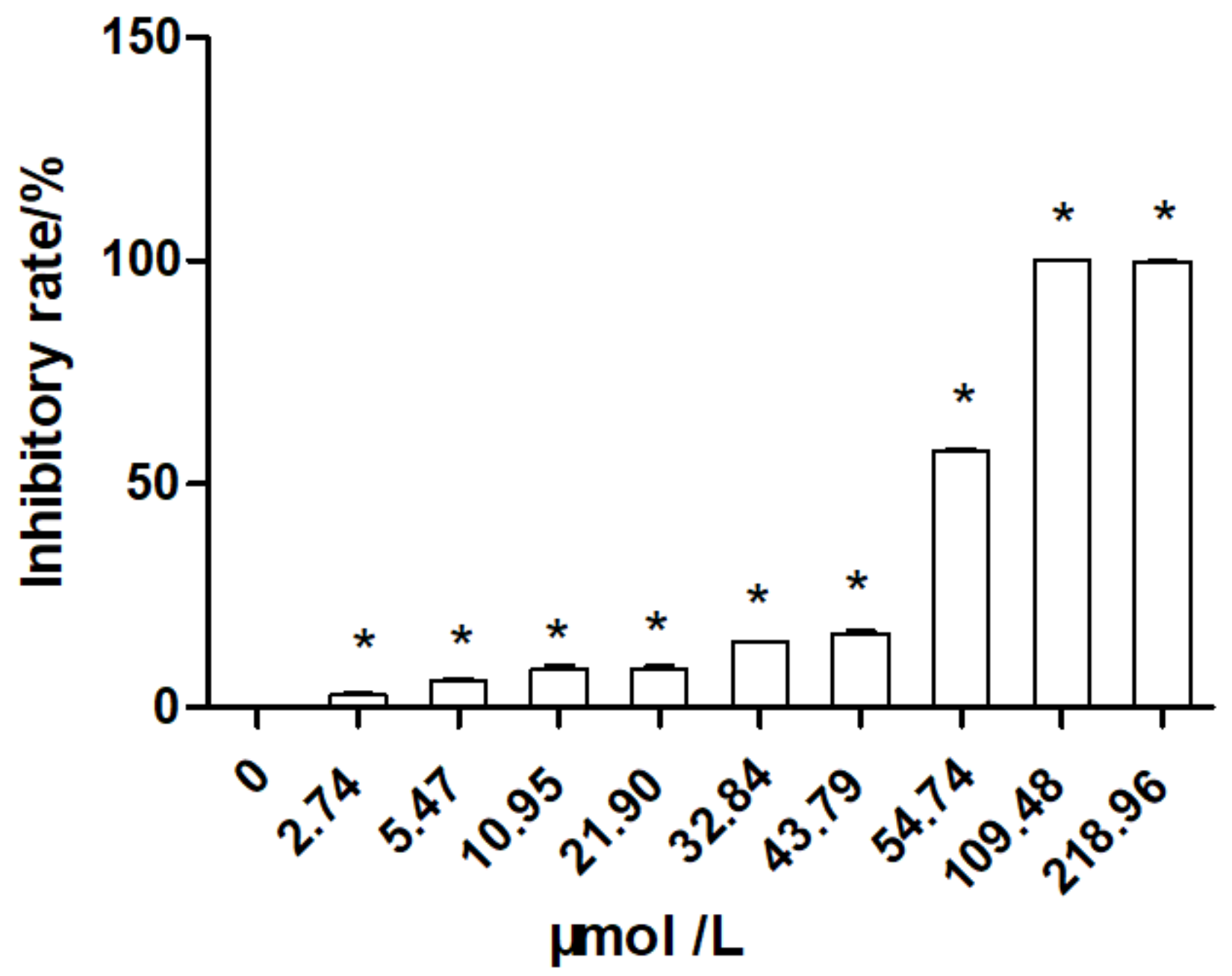

Figure 2

CCK-8 assay to detect the inhibitory effect of OA on the proliferation of U251 glioma cells. Data represent mean $\pm S D$ of three different experiments. ${ }^{*}<<0.05$ compared with control group. 


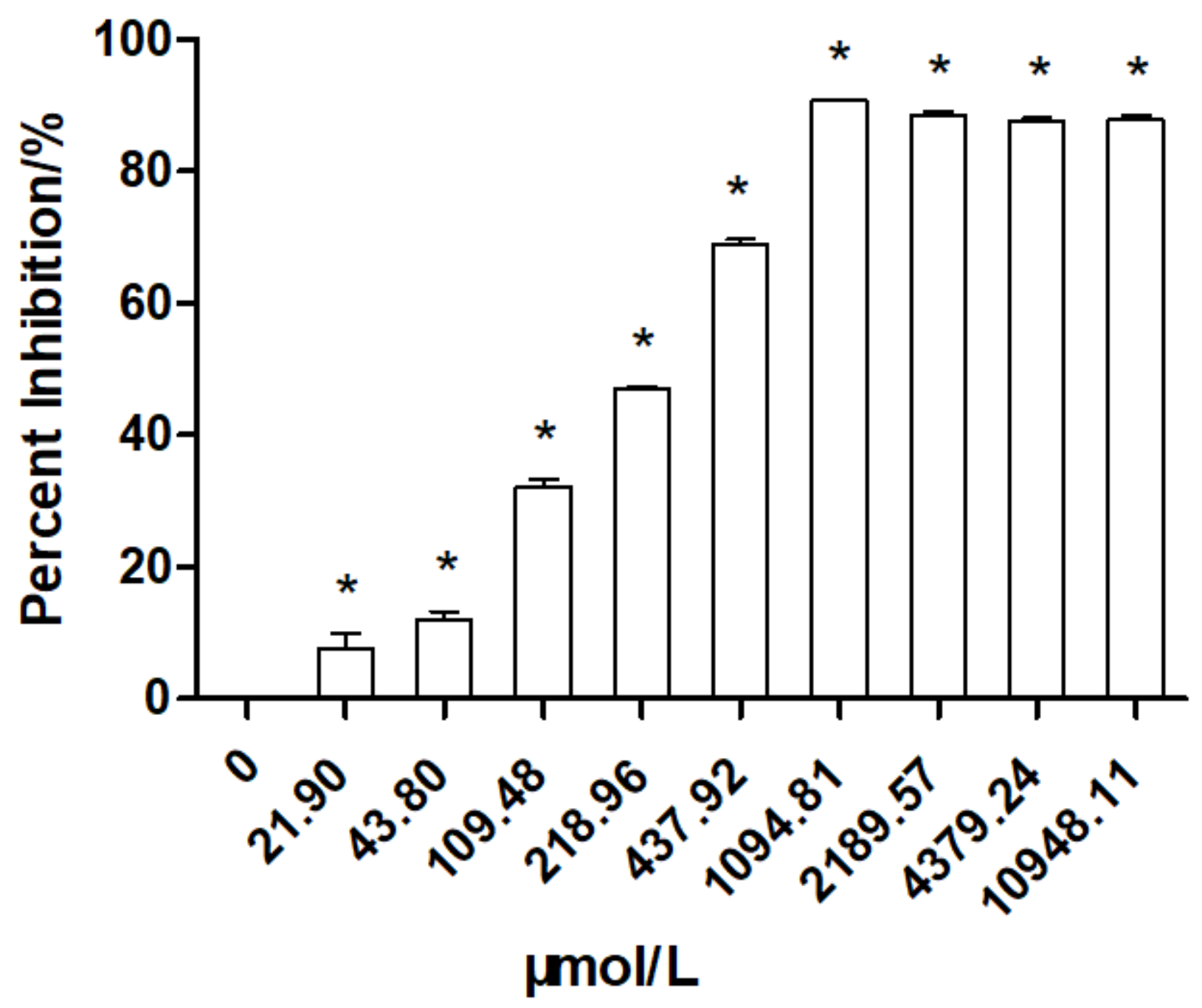

Figure 3

MTT assay to detect the inhibitory effect of OA on the proliferation of U251 glioma cells. Data represent mean \pm SD of three different experiments. ${ }^{*} P<0.05$ compared with control group.

Control

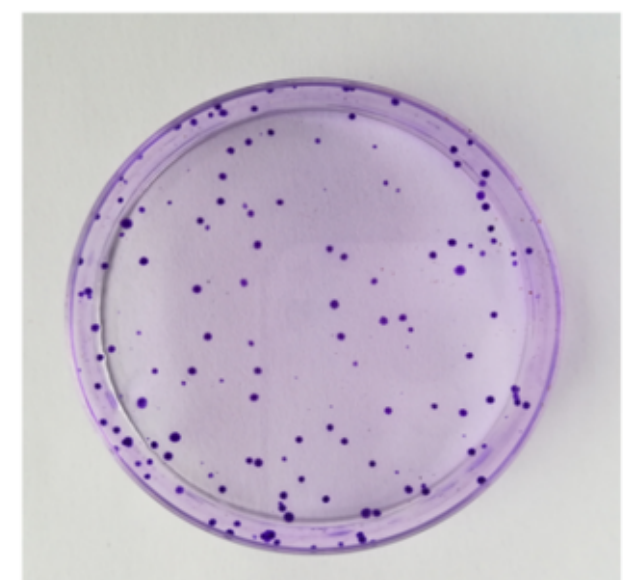

\section{Oleanolic Acid}
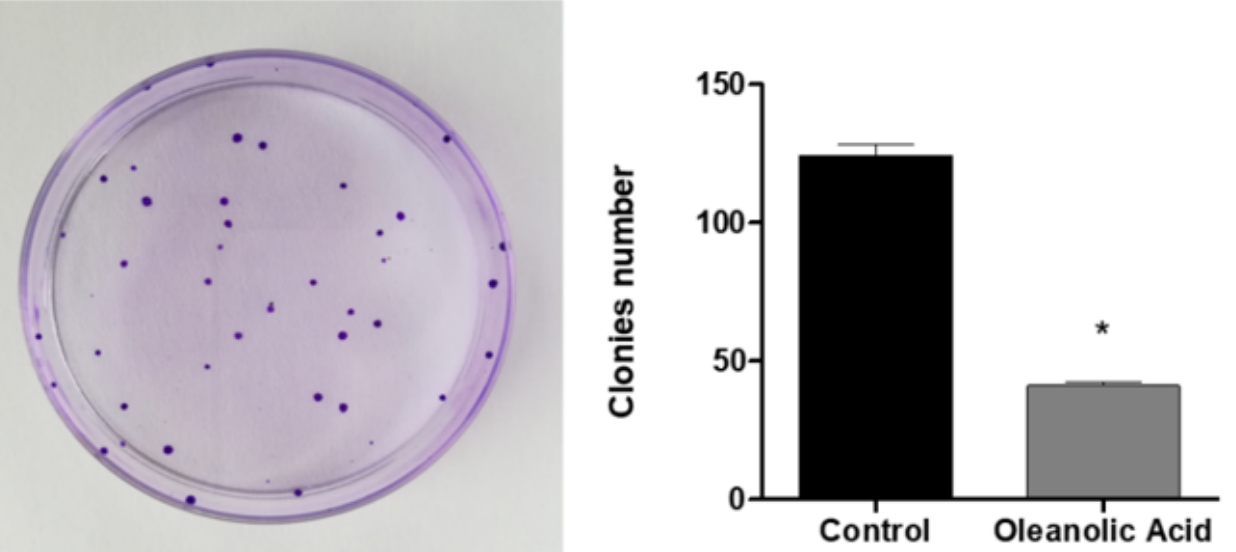
Figure 4

Cell cloning to detect the proliferation inhibitory effect of OA on human glioma U251 cell. Data represent mean \pm SD of three different experiments. ${ }^{*} P<0.05$ compared with control group.

Control

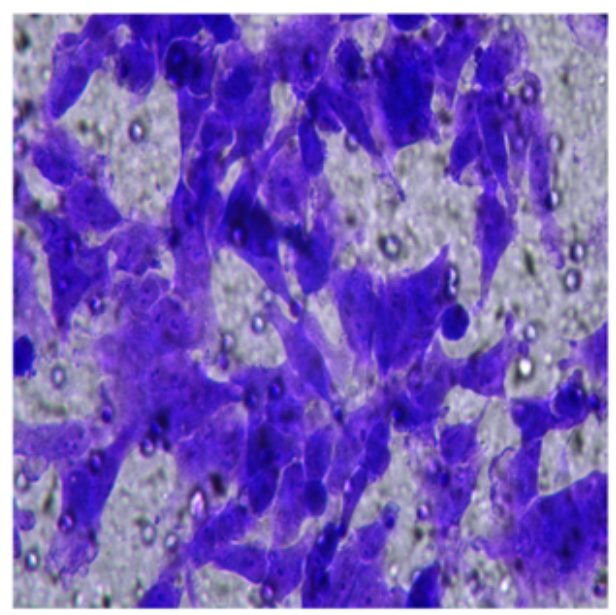

Oleanolic Acid

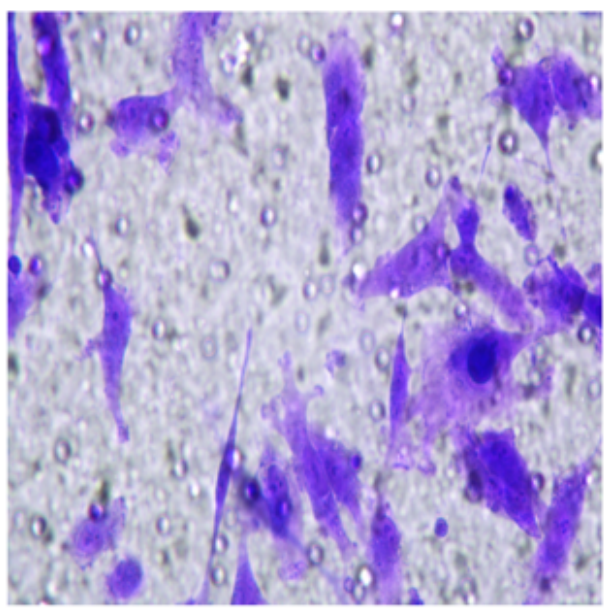

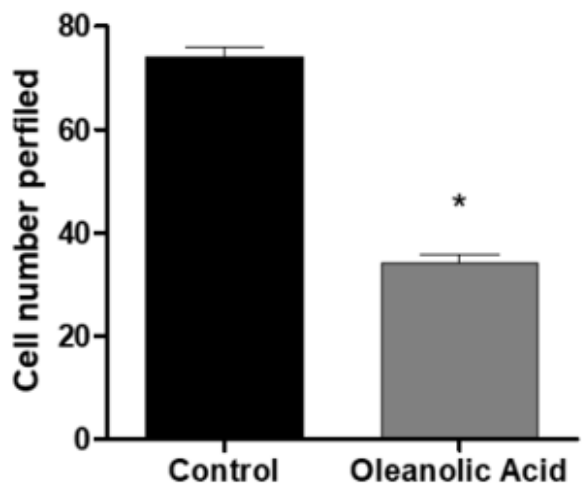

Figure 5

Transwell chamber method to detect the effect of OA on the invasion of human
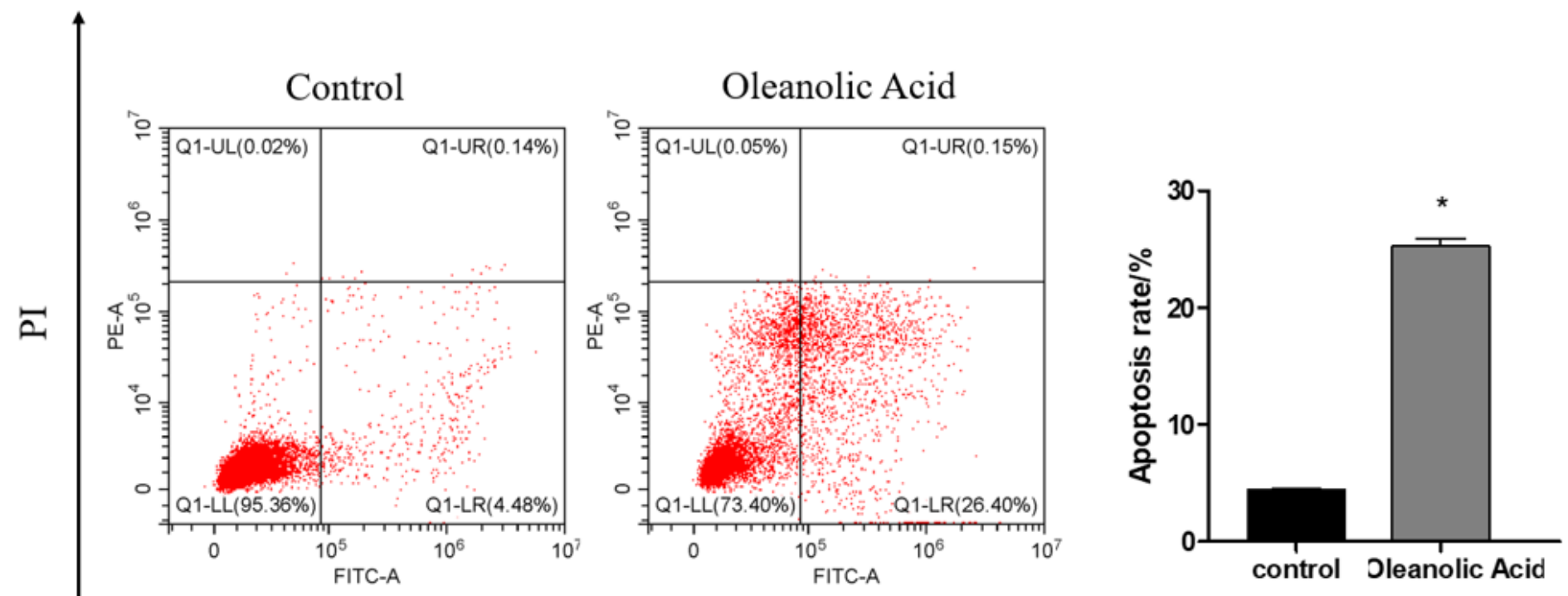

\section{Figure 6}

Annexin $\mathbb{B}$-FITC/PI double staining method to detect the promoting effect of OA on the apoptosis of human glioma U251 cells. Data represent mean \pm SD of three different experiments. ${ }^{*} P<0.05$ compared with control group. 

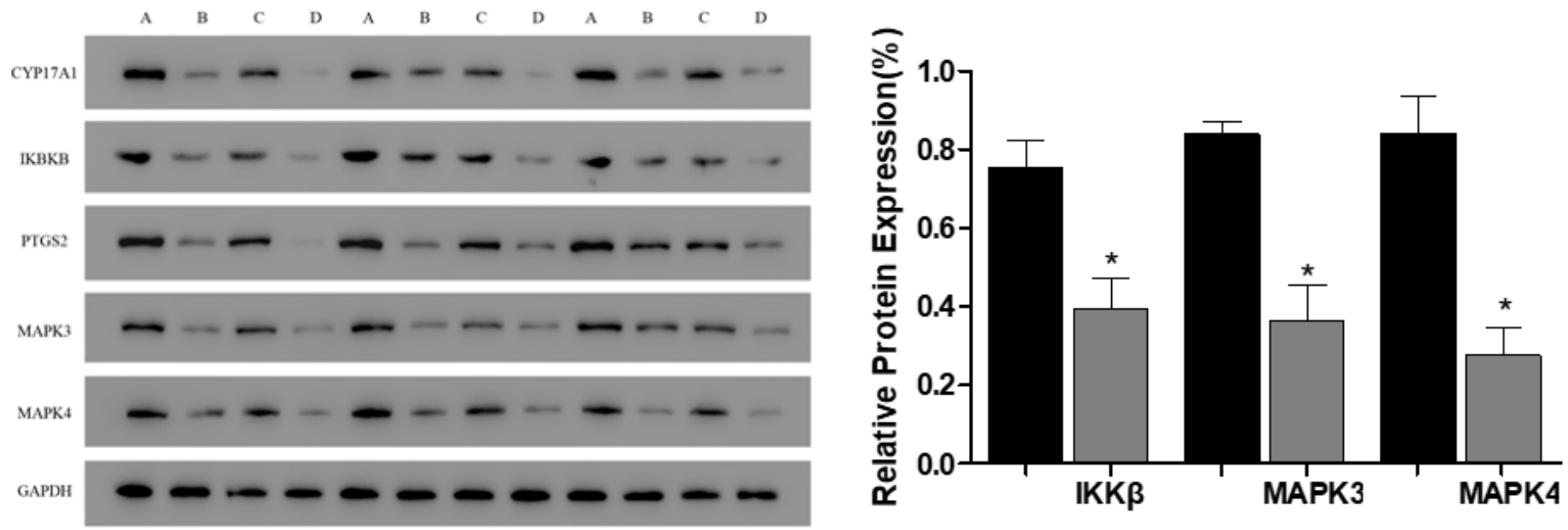

\section{Figure 7}

Western blot detection of OA on the expression of IKK- $\beta$, MAPK3, and MAPK4 proteins in glioma U251 cells. Data represent mean \pm SD of three different experiments. ${ }^{*} P<0.05$ compared with control group. 